\title{
El dispositivo en las ciencias sociales y en la educación: La piedra angular ${ }^{1}$
}

\section{(The dispositive in social sciences and education: The cornerstone)}

\author{
*Luis Peñalver Bermúdez \\ Universidad Pedagógica Experimental Libertador, Venezuela \\ *Email de correspondencia: luisrafaelpenalver@gmail.com
}

\section{RESUMEN}

En este apartado se pretende realizar una aproximación crítica de las ciencias sociales y de la educación en el presente, fundamentada en una evaluación retrospectiva que se apoya en el dispositivo, "herramienta" fundamental en el pensamiento foucaultiano. A lo largo de trabajo, se promueve la reflexión teórica-metodológica sobre las formas de pensar y representar las ciencias sociales y la educación a través de la historia. Finalmente, a modo de conclusión se determina que las ciencias sociales y la educación han demostrado un avance significativo tanto en lo epistemológico como en lo teórico-metodológico. Desde hace ya varios años, la concepción, planificación y construcción del discurso ha experimentado la influenciada de nuevas corrientes metodológicas y campos investigativos propuestos por individuos que se han convertido en cómplices teórico-metodológicos de las ciencias sociales y la educación; entre ellos, Michel Foucault, quien ha contribuido a estas áreas a través de la proposición del concepto del dispositivo, como mecanismo para descubrir y exponer sistemas de ideas y pensamientos en las ciencias sociales y en la educación.

Palabras clave: dispositivo; foucault; discurso; ciencias sociales; educación.

${ }^{1}$ Un préstamo teórico de Castro Orellana (2008), contiene el rasgo vertebral del título que precede a este Capítulo: “Así como el estudio de las reglas que caracterizan las formaciones discursivas, encuentran en la noción de episteme su pilar de apoyo, la investigación sobre la conexión entre prácticas discursivas y prácticas extra discursivas, tiene en el concepto de dispositivo su piedra angular" (p. 111, negrillas nuestras)

Profesor Titular, jubilado, de la Universidad Pedagógica Experimental Libertador (Venezuela). Profesor en Ciencias Sociales, mención Historia. Especialista y Magister en Investigación de la Educación. Doctor en Educación. Consultor UNESCO-IESAL en el área de formación docente. Correo-e: luisrafaelpenalver@gmail.com 


\begin{abstract}
This work intends to provide a critical approach to social sciences and education at present, based on a retrospective evaluation that supports itself on the dispositive, a fundamental "tool" in the Foucauldian thought. Throughout this work, a theoretical-methodological reflection on the ways of thinking and representing the social sciences and education through history is promoted. Finally, as a conclusion, it is determined that the social sciences and education have demonstrated significant progress both on its epistemological and theoretical-methodological basis. For several years now, the conception, planning, and construction of discourse have been influenced by new methodological trends and research areas proposed by individuals who have become theoretical and methodological collaborators of the social sciences and education. Among these individuals is Michel Foucault, who has contributed to these fields through the introduction of the dispositive as a mechanism to discover and present structures of ideas and thoughts in social sciences and education.
\end{abstract}

Keywords: dispositive; foucault; discourse; social sciences; education.

\title{
50 Cumbres
}




\section{INTRODUCCION}

Peter Burke (1999), en su texto: La revolución historiográfica francesa, ofrece un apretado recorrido de la creación histórica francesa innovadora, que, de manera general, se conoce con el nombre de La nouvelle histoire, que "es por lo menos tan famosa como el francés y tan controvertida como la nouvelle cuisine". (p. 11) En buena medida, los intelectuales que en 1929 dieron a conocer la revista de historia Annales, son responsables directos e indirectos de esta nouvelle histoire. En el núcleo básico de la escuela de los Annales, pueden identificarse a: Lucien Febvre, Marc Bloch, Fernand Braudel, Georges Duby, Jacques Le Goff y, más recientemente, Emmanuel Le Roy Ladurie. Burke indica, que

Cerca del borde se encuentran Ernest Labrouse, Pierre Vilar, Maurice Agulhon y Michelle Vovelle, cuatro distinguidos historiadores cuyo compromiso con un enfoque marxista de la historia... los coloca fuera del círculo interior. En el borde o más allá del borde, están Roland Mousnier y Michel Foucault (Burke,1999, p. 11).

Foucault (1926-1984), en el ámbito de las creaciones intelectuales francesas relacionadas con las ciencias sociales y la educación, irrumpe contra la tradición "continuista" de escribir la historia e inicia trabajos de investigación con y desde nuevas categorías como micropolítica, arqueología, genealogía, dispositivo, entre otras, abriendo posibilidades de investigación en campos inexplorados o no explorados suficientemente, como los sistemas de pensamientos.

En la Introducción al texto de Foucault (1988): Un diálogo sobre el poder y otras conversaciones, Miguel Morey expone un esquema que le permite dar cuenta del itinerario intelectual de Foucault, que ha dado "lugar a dos propuestas metódicas: el método arqueológico y el método genealógico" (p. iii). Desde esta misma orientación, la periodización de sus principales textos, permitiría establecer tres grandes grupos: primero, la Formación del método; segundo, el Método arqueológico y, tercero, el Método genealógico. En cuanto a las obras del período denominado Método arqueológico, Morey indica, que aquí "ocupa un lugar central la preocupación por la política del discurso" (p. iii). Sobre el Método genealógico, señala: "Se iniciaría con el artículo Nietzsche, la Génealogie, l'Histoire (1971), en el que se sientan las bases de su modo actual de entender la historia" (p. iii-iv). En otra perspectiva, Jalón (1994), alcanza a proponer, con mucha cautela, lo que denomina "estilo arqueogenealógico", y señala que "Su objetivo es, pues, delimitar la aparición, la regularidad y las condiciones de posibilidad de un determinado tipo de discurso" (p. 65). Aun así y con todo lo que ello implique, también conveniente acercarse, directamente, a lo que Michel Foucault dice al respecto.

Si se le preguntase a Foucault (2000) ¿Cómo es la genealogía?, diría en principio: "La genealogía es gris, es meticulosa y pacientemente documentalista. Trabaja sobre sendas embrolladas, garabateadas, muchas veces reescritas" (p. 7). Pero una interrogante con mayor intensidad, sería la que preguntaría por la genealogía misma: ¿Qué es hacer genealogía?:

Hacer la genealogía de los valores, de la moral, del ascetismo, del conocimiento, [cंde las ciencias sociales, de la educación?] no será por tanto partir en la búsqueda de su "origen", minusvalorando como inaccesibles todos los episodios de la historia; será por el contrario ocuparse en la meticulosidades y en los azares de los comienzos; prestar una escrupulosa atención a su derrisoria malevolencia; prestarse a verlas surgir quitadas las máscaras, con el rostro del otro; no tener pudor para ir a buscarlas allí donde están -revolviendo los bajos fondos- dejarles el tiempo para remontar el laberinto en el que ninguna verdad nunca jamás las ha mantenido bajo su protección... Es preciso saber reconocer los sucesos de la historia, sus sacudidas, sus sorpresas, las victorias afortunadas, las derrotas más digeridas, que dan cuenta de los comienzos, de los atavismos y de las herencias; como hay que saber diagnosticar las enfermedades del cuerpo, los estados de debilidad y energía, sus trastornos y sus resistencias... (Foucault, 2000, pp. 11-12).

Desde el aliento foucaultiano por las posibilidades de la genealogía o con la perspectiva del "estilo arqueogenealógico", según la propues- 
ta de Jalón, está una oportunidad metodológica para indagar el conjunto de reglas, de principios; el ordenamiento, la organización de los procedimientos que engendran, ponen en movimiento, determinan las normas de los enunciados como representaciones de saber, para hacer un trabajo como arqueogeneaólogo sobre esa especie de genealogía cognitiva que se sucede en capas discontinuas en las ciencias sociales y en la educación, en una especie de descubrimiento de los datos de saber que están en las distintas estratigrafías del conocimiento; capas, con determinadas características que también se integran con conjuntos previos y posteriores, se entrecruzan en el tiempo, en las épocas.

El análisis arqueogenealógico, o propiamente genealógico, de la composición atiborrada de trozos de ideas, de conceptualizaciones, de inquietudes discursivas, de representaciones epistémicas, "no busca hacer aparecer la verdad de nuestro pasado, sino de mostrar el pasado de nuestras verdades" (Morey, 1983, p. 23). Se trata de una oportunidad indagatoria para comprender el presente y explicar cómo y por qué ciertos saberes emergen y se configuran. Esto en clave de las ciencias sociales y de la educación, ya luce atractivo. No sería, en términos tradicionales, investigar el pasado de las ciencias sociales y de la educación; sino, en otros términos, indagar el presente de las ciencias sociales y de la educación, a través de su pasado.

No se trata, en lo absoluto, de buscar los orígenes, perdidos o borrosos, sino la formación en lo nuevo, la emergencia, eso que Foucault llamó la actualidad, aunque Nietzsche lo haya llamado lo inactual, lo intempestivo. Sería intentar sacar a la luz los sistemas de saber que se han estructurado en las ciencias sociales y en la educación, descubriendo sus constituciones históricas; por cierto, sistemas y constituciones que siguen "vigentes" y en los que se sigue aferrados. En el fondo, se aspira diseñar una crítica de las ciencias sociales y de la educación en el presente, fundada en un análisis retrospectivo, con apoyo de una "herramienta" fundamental en el pensamiento foucaultiano, denominada dispositivo.

\section{CONTEXTO}

Desde el presente apartado, en términos generales, se aspiran promover reflexiones teórico-metodológicas sobre las formas de pensar y representar, históricamente, las ciencias sociales y la educación. Una perspectiva definida en estos ángulos, pudiera llamar la atención, al menos sobre cuatro componentes sustantivos.

En primer lugar, no se daría mucha atención a recorridos histórico-lineales, de tendencia interpretativa-explicativa, determinantemente causales y marcadamente cronológicos.

En segundo lugar, los esfuerzos de investigación, tendrían sus énfasis en intentar analizar los modos de ver, de hablar y de representar las ciencias sociales y la educación y, en tanto configuran regímenes de verdad que van fraguando y configurando lo que se dice, piensa y hace de ellas y con ellas, en distintos momentos de la historia.

En tercer lugar, tantear los impulsos de una perspectiva de investigación completamente diferente, para atender lo que históricamente ocurre en las ciencias sociales y en la educación, implica otros modos de interrogación, otros mecanismos interpelativos, que permitan diseñar un sistema óptico distinto, para aprehender pistas de visibilidad y rasgos de enunciabilidad.

En cuarto lugar, redefinir rumbos de reapropiaciones epistemológicas desde el pensamiento de Michel Foucault, propiamente como rupturas epistemológicas, también significa reflexionar sobre componentes de su obra y reinventarlos para pensar, en el presente caso, las ciencias sociales y la educación. Desde aquí, se despliega entonces una invitación relacionada con el asunto de las "cajas de herramientas", de manera que todo investigador interesado en la obra de Foucault, al decir de él mismo, pueda "abrirlas, servirse de una frase, de una idea, de un análisis, como si se tratara de un destornillador o de unos alicates para hacer cortocircuitos, descalificar, romper los sistemas de poder" (Foucault, 1991a, p. 88).

Una aclaratoria con carácter previo. Las notas del presente Capítulo, están concebidas para reflexionar sobre aspectos del pensamiento foucaultiano y sus relaciones con las ciencias socia- 
les y la educación, particularmente en lo que se refiere a la herramienta dispositivo, pero, y este es un pero muy importante, seguro estoy que los investigadores escribirán sobre las ciencias sociales y la educación y también sobre temas muy particulares. Pero no se puede desaprovechar la oportunidad de leer, escribir y pensar sobre los mismos investigadores, sobre sus ideas y así, quizás, con Marc Auge (1998), se pueda saber "en qué dirección alzan el vuelo, a que otros pensamientos se unen y en que palabras se refugian" (p. 12)

\section{LA HISTORIA}

En 1968, Jean-Pierre El Kabbach tuvo la oportunidad de realizar una breve entrevista a Michel Foucault, que luego fue publicada en español en el libro Saber y verdad, en 1991b.

Sin ningún miramiento, El Kabbach lanza una pregunta con mucho espesor: "Sartre le reprocha a Ud. y a otros filósofos, el minusvalorar y menospreciar la historia ces cierto?" La Respuesta de Foucault, quizás nada grata para algunos profesionales de las ciencias sociales, está argumentada para enfrentar un tipo de historia que "es una especie de grande y tosca continuidad en la que se engarzan la libertad de los individuos y las determinaciones económicas o sociales". Más tarde, Veyne (1984), admirador del pensamiento de Foucault, en un tono conciliatorio, dice que "lo único que dice Foucault a los historiadores es: se puede seguir explicando la historia como se ha explicado siempre, pero cuidado: si se mira detenidamente, haciendo abstracción de los tópicos, se advierte que hay más cosas que explicar de lo que se pensaba; hay formas extrañas que han pasado inadvertidas" (p. 210).

En Las palabras y las cosas (1999), Foucault se opone a una historia como línea de continuidad, como evolución progresiva y el estudio de los saberes se convierte ahora en campo de batalla, poniendo énfasis en tratar de descubrir las condiciones históricas que hicieron posible la construcción de esos saberes ${ }^{3}$ (Cf.: Sanden y González, 1997, p. 5).

En el dial de las investigaciones ligadas a las ciencias sociales a y la educación, hay, entonces, oportunidad para una historia que haga posible dar cuenta de la emergencia de los juegos de verdad; en suma, la historia crítica del pensamiento no es ni una historia de las adquisiciones de la verdad ni de sus ocultaciones; es la historia de las emergencias de los juegos de verdad. Es la historia de las 'viridicciones', entendidas como las formas según las cuales, los discursos capaces de ser llamados verdaderos o falsos, son articulados con un dominio de las cosas: cuáles son las condiciones de este surgimiento; qué precio se ha pagado por ello y cómo fue; qué efectos ello ha provocado en lo real y el modo en el cual, vinculando un cierto tipo de objeto con ciertas modalidades de sujeto, se ha constituido durante un tiempo, un espacio y, en unos individuos particulares, el a priori histórico de una experiencia posible (Florence ${ }^{4}, 1996$, pp. 4-5)

Para el caso probable de una historia crítica de las ciencias sociales o de la educación de alguno de sus temas más particulares, las interrogantes precedentes son de una importancia suprema. Ahora, hay un aspecto que, por su volumen, debe destacarse: ¿Cómo los individuos son convertidos en sujetos? ¿Cómo en los procesos educativos y en las construcciones discursivas de las ciencias sociales, "entran" individuos y "salen" sujetos?: el personaje central, el que viene representado (y represado) en los grandes relatos del occidente moderno, ya no está ${ }^{5}$.

${ }^{3}$ Imaginar esto en clave de ciencias sociales y educación, es un todo un atractivo reto.

${ }^{4}$ Maurice Florence es un seudónimo tras el que están el mismo Michel Foucault y François Ewald, quien fue su secretario privado.

${ }^{5}$ En unas notas realmente contundentes, Vidal (1999) expresa lo siguiente: "si algo queda por hacer al historiador, esto será la articulación de una prospección genealógica que sirva para desmontar los mecanismos disciplinares de identificación, clasificación y procesamiento de los integrantes de unas sociedades humanas encerradas en sus propios discursos" (p. 7). Ver, además: Foucault, 1991a, p. 56 y Jalón, 1994, pp. 32.33. 


\section{LOS DISPOSITIVOS}

Uno de los libros donde abundan las explicaciones sobre los dispositivos, es el identificado con el título de Saber y verdad, donde -a propósito del lanzamiento editorial del texto Historia de la sexualidad, Foucault (1991a) es interrogado, precisamente, sobre el dispositivo de sexualidad, respondiendo con varias ideas:

1. En primer lugar, el dispositivo identifica "un conjunto... heterogéneo que comprende discursos, instalaciones arquitectónicas, instituciones, decisiones reglamentarias, leyes, medidas administrativas, enunciados científicos, proposiciones filosóficas, morales, filantrópicas... El dispositivo es la red que puede establecerse entre estos elementos" (pp. 128-129).

2. En segundo lugar, señala luego, que el dispositivo es la "naturaleza del vínculo que puede existir entre estos elementos heterogéneos" (ídem)

3. Su tercera afirmación se refiere a que se trata de una "especie de formación que, en un momento histórico dado, tuvo como función mayor la de responder a una urgencia” (ídem).

4. En cuarto lugar, un dispositivo siempre se encuentra inscrito en un juego de poder, soportando unos tipos de saber.

Ahora, atrevidamente, si desde la noción de dispositivo se intentara realizar una elemental operación crítica en el ámbito de los pensamientos de las ciencias sociales y de la educación, y sus historias respectivas, grosso modo pudieran hacerse las siguientes interrogantes:

1. ¿Cuál es ese conjunto heterogéneo, diverso múltiple, que identifica en un momento dado, el dispositivo en las ciencias sociales o en lo educativo? ¿Cómo es esa red (léase dispositivo)? que pudiera reconocerse entre los componentes del conjunto heterogéneo? En determinado momento ¿qué discurso, cuáles edificaciones, cuáles instituciones, reglamentaciones, leyes, conocimientos científicos, filosofías, moralidades, forman el dispositivo de las ciencias sociales o educativo? De otra manera: ¿cómo están pensadas las ciencias sociales? ¿cómo está pensada la educación? Al margen: ¿cómo desde ciertos autores, desde sus enunciabilidades educativas o sobre las ciencias sociales, pueden iniciarse procesos para definir el dispositivo en la educación o en las ciencias sociales? ¿En qué medida, tales ideas sobre la educación o las ciencias sociales, "marcan" un componente de ese conjunto heterogéneo? Pero también ¿̇cómo el pensamiento de un autor o entidad, se hace transversal en el pensamiento educativo o de las ciencias sociales? En su tiempo y en el presente constituyéndose.

2. También puede acudirse a otras preguntas: ¿A cuál urgencia tenía que responder o respondía tal o cual autor? ¿Cómo se había formado tal emergencia? ¿En qué juego de poder estaba anotado el pensamiento educativo o de las ciencias sociales, expresado? ¿Qué tipos de saber soportaba y cuáles no? ¿Qué dirían los pensamientos educativos y sobre ciencias sociales, de autores nacionales, latinoamericanos, desde las inquietudes que pudieran desatar los análisis de los dispositivos?

Aun cuando a estas alturas lo relacionado con el dispositivo, en términos generales, pareciera que cubre todos sus referentes conceptuales y metodológicos, no hay que olvidar que el mismo Foucault (1991a), llegó a señalar lo siguiente: "Respecto al dispositivo, me encuentro ante un problema del que todavía no he conseguido salir" (p. 130). Eso, quizás, es lo mejor y más admirable de su estilo: no hay "metodologías", agendas, cartas procedimentales estructuradas y preconcebidas. Por eso, la denominación de "operación crítica”, puede ser un buen punto de reflexión y discusión.

Un abordaje exquisito sobre dispositivo, es el que hace Deleuze (1999), quien se pregunta y responde: “¿Qué es un dispositivo? En primer lugar, es una especie de ovillo o madeja, un conjunto multilineal. Está compuesto de líneas de diferente naturaleza y esas líneas siguen direcciones diferentes, forman procesos siempre en desequilibrio y esas líneas tanto se acercan unas a otras como se alejan unas de otras, Cada línea está 
quebrada y sometida a variaciones de dirección, sometidas a derivaciones" (p. 115)

Siguiendo a Deleuze (ídem, pp. 155-156), las dos primeras dimensiones de un dispositivo, son las curvas de visibilidad y las curvas de enunciación. La visibilidad, primera dimensión, está hecha de líneas de luz; según como se desplieguen, pueden distribuir lo visible y lo no visible. El régimen de enunciación, segunda dimensión, es el que distribuye las posiciones diferenciales de sus elementos, expone variables, es lo que, en definitiva, permite definir el dispositivo.

"En todo dispositivo" dice Deleuze (1999), hay que distinguir lo que somos (lo que ya no somos) y lo que estamos siendo: la parte de la historia y la parte de lo actual. La historia es el archivo, la configuración de lo que somos y dejamos de ser, en tanto que lo actual es el esbozo de lo que vamos siendo", y más adelante: "En todo dispositivo debemos desenmarañar y distinguir las líneas del pasado reciente y las líneas del futuro próximo, la parte del archivo y la parte actual, la parte de la historia y la parte del acontecer, la parte de la analística y la parte del diagnóstico (pp. 159-160).

Este conjunto de ideas acerca del dispositivo, permitirá hacer un boceto de -al menos- seis breves ideas:

1. La primera: el dispositivo es una red. No hay espacio para considerar el dispositivo como una dimensión aislada, autárquica en sí. La noción misma de red implica un entrelazamiento multiforme, atravesada de desigualdades, como una especie de conjunto de vías, pero sin dirección homogénea o regular; que ofrece más bien una organización con diferentes ramificaciones intervinculadas en caos permanente. Pareciera que el dispositivo es una trama de despliegue variado, con opción permanente y constante de redireccionarse. El dispositivo, abusando del descubrimiento de Michael Barnsley, parece una especie de fractal, sobre todo en lo concerniente a lo de la estructura compleja (en el sentido morineano) y a la imagen. Un dispositivo es un conjunto de representación.

2. La segunda: el dispositivo es la naturaleza del vínculo. Un elemento, aislado, solitario, no hace al dispositivo. Un componente de la red no es la misma red. Así de sencillo. El dispositivo es la esencia misma de los vínculos.

3. La tercera: el dispositivo responde a una urgencia. El dispositivo es gestado, formado, constituido y su despliegue responde a un momento histórico dado, a una necesidad apremiante. Por eso el dispositivo es una especie de procedimiento de urgencia, para atender una situación determinada.

4. La cuarta: el dispositivo está anotado en un juego de poder. El asunto del poder es uno de los aspectos más atractivos de Foucault. El dispositivo, al soportar unos tipos de saber, al incluir y excluir saberes, al indicar solo ciertos saberes, representa también un juego de poder, que también se ejerce sobre "el alma (soberana del cuerpo, sometida a Dios), la conciencia (soberana en el orden del juicio, sometida al orden de la verdad), el individuo (soberano titular de sus derechos, sometido a las leyes de la naturaleza o a las reglas de la sociedad), la libertad fundamental (interiormente soberana, exteriormente consentidora y adaptada a su destino" (Foucault, 1980, p. 34).

5. La quinta: el dispositivo tiene curvas de visibilidad. En el dispositivo hay líneas de luz, pero la luz permite una doble dimensión: lo que es visible y lo no visible. Hay cosas que, desde el dispositivo, se pueden ver y otras no; pero no se trata de que las últimas sean invisibles en todo momento, sino, que ciertos biombos de oscuridad impiden que sean vistas; las líneas de luz están en otro lado.

6. Y la sexta: el dispositivo tiene curvas de enunciación. Las curvas de enunciación se refieren a aquello que es expuesto, exhibido, formulado. Esa vertiente enunciativa se aprecia en la distribución de elementos, de variables, que contiene el dispositivo. Las curvas de enunciación, que son transversales al dispositivo, se destacan como componentes para la definición. El dispositivo se enuncia (es enunciado) de una forma peculiar, específica. 


\section{DISPOSITIVOS ESPECIFICOS}

El componente metodológico sustantivo en las investigaciones que en la educación y las ciencias sociales pudieran adelantarse, no se identificará, exclusivamente, afinando los términos teóricos para saber lo que el dispositivo significa. Ayudaría mucho, si esta responsabilidad preliminar se enriquece con la detección de ciertos dispositivos específicos, a manera de ejemplos. En esta oportunidad, se disponen cuatro: a) de enseñanza mutua; b) de subjetividad; c) de Iglesia y d) el de la gubernamentalidad, leído en clave de crisis del capitalismo.

- Luego de una extensa exposición, que bien pudiera conectarse con la experiencia del sistema lancasteriano en América Latina, en pleno desarrollo de las Guerras de Independencia, dice Foucault (2005b):

Tenemos con esto el esbozo de una institución de tipo 'de enseñanza mutua', donde están integrados en el interior de un dispositivo único tres procedimientos: la enseñanza propiamente dicha, la adquisición de conocimientos por el ejercicio mismo de la actividad pedagógica y, finalmente, una observación recíproca y jerarquizada (pp. 181).

- Un segundo dispositivo específico, se refiere al dispositivo de subjetividad, o como lo aclara el autor: "la historia de las relaciones entre sujeto y verdad". Expone:

Y diré que quien quiera hacer la historia de la subjetividad -o, mejor, la historia de las relaciones entre sujeto y verdad- debería tratar de recuperar la muy prolongada, muy lenta transformación de un dispositivo de subjetividad, definido por la espiritualidad del saber y la práctica de la verdad por el sujeto, en otro dispositivo de subjetividad que es el nuestro, gobernado, me parece, por la cuestión del conocimiento del sujeto por sí mismo y su obediencia a la ley (Foucault, 2005a, p. 299).

- El dispositivo Iglesia, es el tercero que se logró ubicar, relacionado con la categoría de pastorado:

En rigor, habría que decir..., que el pastorado comienza con un proceso que es absolutamente único en la historia y del que no se encuentra ningún ejemplo en ninguna otra civilización: un proceso por el cual una religión, una comunidad religiosa, se constituyó como Iglesia, es decir, como una institución con pretensiones de gobierno de los hombres en su vida cotidiana, so pretexto de conducirlos a la vida eterna en el otro mundo, y esto a escala no solo de un grupo definido, no solo de una ciudad o Estado, sino de la humanidad en su conjunto. Una religión que pretende de ese modo alcanzar el gobierno cotidiano de los hombres en su vida real con el pretexto de su salvación y a escala de la humanidad: eso es la Iglesia... Creo que con esta institucionalización de una religión como la Iglesia se forma... un dispositivo de poder sin paralelo en ningún otro lugar, y que no dejó de desarrollarse y afinarse durante 15 siglos, digamos desde el siglo II o III hasta el siglo XVIII (Foucault, 2008, p. 150).

- Un cuarto dispositivo específico es el de la gubernamentalidad, vinculado con la crisis del liberalismo y de la economía capitalista:

"Podemos encontrar las crisis del liberalismo en conexión con la crisis de la economía del capitalismo. Podemos encontrarlas, asimismo, cronológicamente desfasadas de esas crisis, y de todas formas su manera de manifestarse, la manera de manejarlas, las reacciones que suscitan los reordenamientos que provocan, no pueden deducirse sin más de las crisis del capitalismo. Es la crisis del dispositivo general de gubernamentalidad, y me parece que podríamos hacer la historia de las crisis de ese dispositivo tal como se introdujo en el siglo XVIII.

Eso es entonces lo que trataré de hacer este año, para lo cual, en cierto modo, tomaré las cosas retrospectivamente, es decir: partir de la manera como, durante los últimos treinta años, se postularon y formularon los elementos de esa crisis del dispositivo de gubernamentalidad e intentaré recuperar, en la historia del siglo XIX, algunos elementos que permiten esclarecer el modo como se experimenta, se vive, se practica y se formula actualmente la crisis del dispositivo de gubernamentalidad (Foucault, 2009, pp. 79-80). 


\section{LOS ALCANCES DE INTERPRETACIÓN}

En esta sección se expondrán creaciones, caracterizadas, precisamente, por su diversidad interpretativa y serán elementos valiosos en los acercamientos y tratamientos que diversos autores dan al tema del dispositivo:

- ¿Qué es un dispositivo? (Deleuze, 1989)

- Cartografías del poder y del saber. (Leal, 1996, pp. 263 y ss)

- El dispositivo (Alliez, 1998, pp. 99-103)

- El método y la analítica del discurso (Páez Díaz, 2002, pp. 115 y ss)

- Despliegue de un dispositivo pedagógico, el encuentro de geografía (Valera-Villegas, 2002, pp. 232 y ss)

- ¿Qué es un dispositivo? (Moro Abadía, 2003)

- El discurso político: rasgos genéricos (Kornblit, 2004, pp. 179 y ss)

- ¿Qué es un dispositivo? (Agamben, 2005, octubre 12)

- Entre la escritura y el deseo: un nuevo espacio de producción (Betancourt, 2006, pp. 51 y ss)

- El dispositivo, instrumento de análisis genealógico (Moro, 2006, pp. 140 y ss)

- De la episteme al dispositivo (Castro Orellana, 2008, pp. 107-115)

- Todo es singular en la historia universal: el discurso (Veyne, 2008, pp. 15 y ss)

- El despliegue de la ontología del presente. (Fortanet, 2010, pp. 95 y ss)

- ¿Qué es un dispositivo?: Foucault, Deleuze, Agamben (García Fanlo, 2011)

\section{CONCLUSIONES}

El escenario epistemológico y teórico-metodológico en los campos de las ciencias sociales y sociales y la educación, ha tomado un avance realmente productivo. Los debates y propuestas en los momentos recientes, por hablar de las tres últimas décadas del siglo XX y la que va del XXI, están llegando cada vez a más grupos y centros de investigación, promoviendo nuevos e inéditos campos de investigación, visiones metodológicas nuevas, al menos para algunas ciencias sociales y la educación y nuevos estilos en la concepción, planificación y construcción del discurso.

Michel Foucault, entre otros que le siguen o adversan, ha sido uno de los mayores cómplices teórico-metodológicos en las ciencias sociales y en le educación. Las historias en claves genealógicas y los estudios de los procesos escolares como escenarios de saber-poder, están allí como buenas muestras.

En este contexto, el dispositivo, como herramienta para descubrir y exponer sistemas de ideas y pensamientos en las ciencias sociales y en la educación, más allá del afán continuista de la "realidad", como aprehensión de especifidades geo-históricas, pudiera ser un cauce para nuevas investigaciones, para otras representaciones discursivas, para otros descubrimientos que permitan exponer "la configuración de lo que somos y dejamos de ser, en tanto que lo actual es el esbozo de lo que vamos siendo", como bien dice Deleuze (ídem).

\section{REFERENCIAS BIBLIOGRÁFICAS}

Agamben, G. (2005, Octubre). ¿Qué es un dispositivo?. Trabajo presentado en la UNLP, La Plata.

Alliez, E. (1998). De la imposibilidad de la fenomenología: Sobre la filosofía francesa contemporánea. Santiago de Cali: Editorial Universidad del Valle.

Auge, M. (1998). Las formas del olvido. Barcelona: Editorial Gedisa.

Betancourt, F. (2006). Historia y lenguaje: El dispositivo analítico de Michelt Foucault. México: Universidad Nacional Autónoma de México

Burke, P. (1999). La revolución historiográfica francesa: La Escuela de los Annales: 19291989. Barcelona: Gedisa Editorial. 
Castro, R. (2008). Foucault y el cuidado de la libertad. Santiago de Chile: Editorial LOM.

Deleuze, G. (1999). Michel Foucault, filósofo. Barcelona: Gedisa.

Florence, M. (1996). Michel Foucault, en Diccionario de Filósofos. Paris: P. U. F.

Fortanet, J. (2010). Foucault y Rorty: presente, resistencia, deserción. Zaragoza: Prensas Universitarias.

Foucault, M. (1980). Microfísica del poder. Madrid: La Piqueta.

Foucault, M. (1988): Un diálogo sobre el poder y otras conversaciones. Madrid: Alianza Editorial.

Foucault, M. (1991a). Historia de la sexualidad. (18va ed.). Madrid: Siglo XXI Editores.

Foucault, M. (1991b). Saber y verdad. Madrid: La Piqueta.

Foucault, M. (1999). Las palabras y las cosas. Una arqueología de las ciencias humanas. México: Siglo XXI Editores.

Foucault, M. (2000). Nietzche, la genealogía y la historia. Madrid: Pre-Textos.

Foucault, M. (2005a). La hermenéutica del sujeto. Madrid: Akal.

Foucault, M. (2005b). Vigilar y castigar: Nacimiento de la prisión. México: Siglo XXI Editores.

Foucault, M. (2008). Seguridad, territorio, población. Madrid: Akal.

Foucault, M. (2009). Nacimiento de la clínica. Madrid: Akal.

García del Pozo, R. (1988). Michel Foucault: un arqueólogo del humanismo. Sevilla: Secretaría de Publicaciones de la Universidad de Sevilla.
García, L. (2011, marzo). ¿Qué es un dispositivo?: Foucault, Deleuze, Agamben. A Parte Rei, Revista de Filosofía, 74, 1-8.

Jalón, M. (1994). El laboratorio de Foucault. Madrid: Antrophos.

Kornblit, A. L. (2004). Metodologías cualitativas en ciencias sociales. Modelos y procedimientos de análisis. Buenos Aires: Editorial Biblos.

Leal, A. (1996). El crepúsculo de la política. Santiago de Chile: Ediciones LOM.

Morey, M. (1983). Lectura de Foucault. Madrid: Taurus.

Moro, O. (2003). ¿Qué es un dispositivo?. EMPIRIA, Revista de Metodología de Ciencias Sociales, 6, 29-46.

Páez Díaz de León, L. (2002). Vertientes contemporáneas del pensamiento social francés. México: Universidad Nacional Autónoma de México.

Sanden, C. \& González, M. (1997). Reformas educativas y estilos de desarrollo. Recuperado de http://fp.chasque.net/ relacion/anteriores/9710/Reforma.html\#serie

Valera-Villegas, G. (2002). Pedagogía de la alteridad. Caracas: UCV.

Veyne, P. (1984). Cómo de escribe la historia. Foucault revoluciona la historia. Madrid: Alianza Editorial.

Veyne, P. (2008). Foucault. Pensamiento y vida. Barcelona: Paidós.

Vidal, R. (1999). La historia y la postmodernidad. Espéculo, Revista de Estudios Literarios, 13. Recuperado de http://pendientedemigracion. ucm.es/info/especulo/numero13/finhisto.html 